\title{
Trends in pre-existing mental health disorders among parents of infants born in Western Australia from 1990 to 2005
}

\begin{abstract}
Melissa O'Donnell PhD, MPysch, GradDipEd NHMRC Early Career Fellow

Denise Anderson

Biostatistician

Vera A Morgan

$\mathrm{PhD}, \mathrm{MSocSc}, \mathrm{BA}$

Professor $^{3}$

Natasha Nassar

$\mathrm{PhD}, \mathrm{MPH}, \mathrm{BEC}$

NHMRC Research Fellow ${ }^{4}$

Helen M Leonard

$\mathrm{MB}$ ChB, MPH

Research Professor

Fiona J Stanley

MD, MSc, FAFPHM

Research Professor

1 Centre for Child Health

Research, University of

Western Australia

Perth, WA.

2 Telethon Institute fo

Child Health Research

Perth, WA.

3 School of Psychiatry

and Clinical Neurosciences,

University of

Western Australia

Perth, WA.

4 Clinical and Population

Perinatal Health Research

Kolling Institute of

Medical Research,

University of Sydney,

Sydney, NSW.

melissao@

ichr.uwa.edu.au
\end{abstract}

MJA 2013; 198: 485-488 doi: 10.5694/mjal2.11783 $\mathrm{t}$ has long been established that parental mental health can affect children's outcomes. ${ }^{1}$ These outcomes not only relate to children's mental health but also language development, behaviour and physical health. ${ }^{2}$ An intergenerational pathway of how parental mental health can adversely affect children's development has been suggested, which includes the direct effects of the illness and associated contextual stressors, such as poverty and disruptions to caregiving. ${ }^{3}$ Social welfare agencies have reported increasing numbers of families facing complex issues, including parental mental health problems and substance use, resulting in concerns about children's wellbeing. ${ }^{4,5}$

While Australia has good data on the rates of mental health disorders in the Australian community, the prevalence of mental health disorders in parents is difficult to estimate. The National Survey of Mental Health and Wellbeing reported that $25 \%$ of individuals aged 16-44 years had a mental health disorder in the 12 months before completing the survey; ${ }^{6}$ this is an age group in which people are most likely to become parents. However data about adults with mental health disorders who are also parents are not routinely collected. To date, no studies have investigated the population prevalence of previous and current mental health disorders in parents, including trends over time. We aimed to fill this research gap by using mental health-related data on public and private hospital inpatient admissions and public outpatient contacts, to provide information on trends in parental mental health disorders in particular diagnostic groups.

\section{Methods}

\section{Study population and data sources}

To determine the population prevalence of parental mental health disorders, we

\section{Abstrac \\ Objective: To determine the prevalence of prior and current mental health disorders in parents, including trends over time.}

Design: A retrospective population cohort study using de-identified linked health data.

Setting: Population of Western Australia.

Subjects: All parents of infants born in WA between 1990 and 2005.

Main outcome measures: Prevalence of prior mental health disorders in parents by birth year and by parent and child characteristics, including Aboriginality, maternal age, socioeconomic status and diagnostic groups.

Results: From 1990 to 2005, there was an increase in prevalence of prior mental health disorders in mothers, from 76 per 1000 births in 1990 to 131 per 1000 births in 2005 (3.7\% increase per year in the odds of children being born to mothers with a prior mental health disorder). There was also a $4.7 \%$ increase in odds per year in the prevalence of mental health contacts that had taken place in the 12 months before the birth year in mothers. In addition, there was an increase in prevalence of prior mental health disorders in fathers, from 56 per 1000 births in 1990 to 88 per 1000 births in 2005 (3.1\% increase in odds per year). The diagnostic group with the highest prevalence in both mothers and fathers was substance-related disorders.

Conclusions: From 1990 to 2005 , there was an increase in prevalence of parents with a prior history of mental health disorders in WA. General practitioners and mental health workers can play an important role in identifying mental illness and in working with families to offer early intervention, referral and support.

conducted a retrospective cohort study of all parents of infants born in Western Australia between 1990 and 2005 using de-identified population level data, linked across health datasets. The health data collections used were WA's Hospital Morbidity Data Collection, Mental Health Information System, Midwives Notification System and Birth Register. The Hospital Morbidity Data Collection contains information on all hospital admissions (public and private hospitals) with corresponding diagnostic information using the International Classification of Diseases (ICD) ${ }^{7}$ coding system recorded for each episode of care for parents from 1970 to 2005 (ICD-8 for 1970 to 1978, ICD-9 for 1979 to June 1999, ICD-10 for July 1999 to 2005). The Mental Health Information System contains information on all mental health-related public and private inpatient admissions and public outpatient contacts for the period 19802005. The Midwives Notification System contains birth information, includ- ing maternal characteristics and infant outcomes, for the period 1990-2005.

Each of the datasets are linked by the WA Data Linkage Branch using probabilistic matching that compares identifiers across datasets with extensive clerical review to resolve doubtful links. ${ }^{8}$ Only a unique project identifier and the individual's clinical information is provided to the researcher; identifying information is removed.

\section{Ethics}

Ethics approval for this study was obtained from the University of Western Australia Human Research Ethics Committee, WA Department of Health Human Research Ethics Committee and Western Australian Aboriginal Health Ethics Committee.

\section{Parental mental health and demographic variables}

Mental health contact by parents was based on any reported diagnosis relating to mental health before the birth 


\section{Prevalence of children having a parent with a prior mental health disorder or current mental health} contact (all birth and first births, Western Australia, 1990-2005)
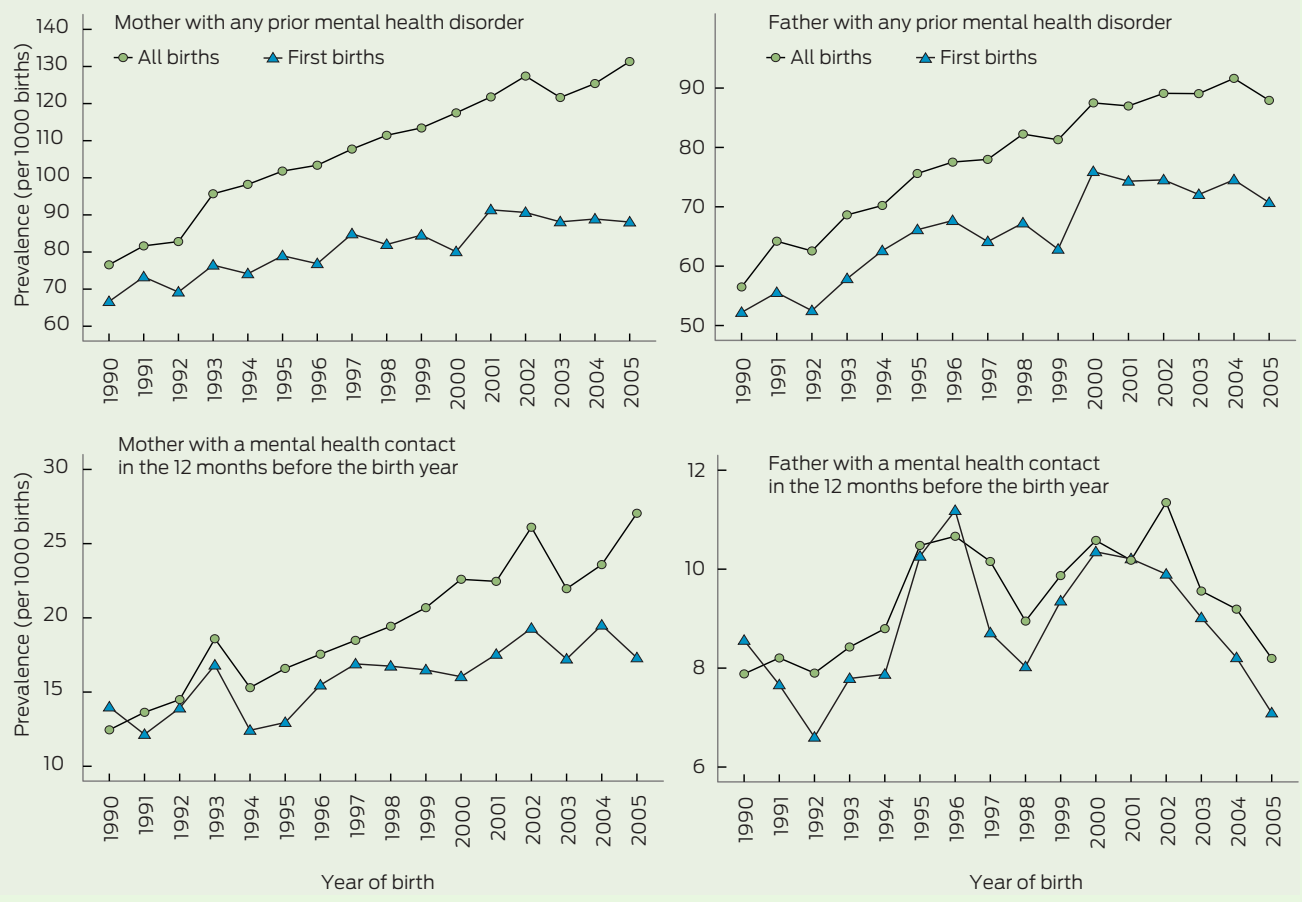

year of the child. Parents were identified as having had a mental health-related hospital admission or outpatient contact if they had mental health-related ICD diagnostic codes (ICD-9 codes 290-319) or external cause of self-harm (ICD-9 codes E950-E959). ${ }^{7}$ ICD-8 and ICD-10 codes were mapped to ICD-9 codes. Parents were defined as having a current mental health contact if there had been a contact in the 12 months before the year of birth. For each birth, parents with a prior mental health contact were counted once even if they had had more than one prior mental health contact; similarly, parents with a current mental health contact were counted once even if they had had more than one mental health contact in the 12 months before the birth year. Mental health diagnostic groups were determined by using the mental health diagnoses reported for the most recent contact before the birth year. If there was more than one diagnosis, the parent was grouped into each relevant diagnostic group. Diagnostic groups and codes for each group are listed in the Appendix (online at mja.com.au).

Aboriginal and Torres Strait Islander children and parents (hereafter referred to as "Aboriginal") were identified if the child or parent was listed in the birth or midwives data as being Aboriginal or Torres Strait Islander. Socioeconomic status was determined using the Index of Relative Social Disadvantage assigned to each collection district by the Australian Bureau of Statistics. ${ }^{9}$ Four levels of disadvantage were classified: 1 (low disadvantage) to 4 (high disadvantage).

\section{Statistical analysis}

Prevalence of prior mental health disorders in parents of children born during the period 1990-2005 (per 1000 births) was calculated for each birth year, using birth data as denominators. Separate analyses were conducted for mothers, fathers, all births, first births and mental health diagnostic groups. First births for mothers were defined as those for which the midwives data listed parity as zero and listed the sum of previous live births and still births as zero. For fathers, first births were identified by selecting the earliest birth. Age-specific rates were calculated for parental age.

Trends are presented as the percentage change in the odds for each increase in birth year and their 95\% CI. Generalised estimating equations were used to calculate robust standard errors for odds ratios when analysing all births, owing to clustering of births to the same parent. Logistic regression was used to calculate odds ratios for Aboriginality, maternal age and socioeconomic status where the outcome was a first-born child who had a mother with a prior mental health disorder, with estimated odds ratios stratified by birth-year group (1990-1993, 1994-1997, 1998-2001 and 2002-2005). $\mathrm{R}$ version 2.11.1 ( $\mathrm{R}$ Foundation for Statistical Computing $)^{10}$ was used for all analyses.

\section{Results}

Of 404022 live births in the period 1990-2005, 43700 children were born to mothers with a prior mental health disorder. Overall, prevalence of prior mental health disorders in mothers increased, from 76 per 1000 births in 1990 to 131 per 1000 births in 2005 (Box 1). There was an estimated 3.7\% (95\% CI, $3.5 \%-4.0 \%$ ) increase per year in the odds of children being born to mothers with a prior mental health disorder. Prior mental health disorders in mothers of first-born children also increased, from 66 per 1000 births in 1990 to 88 per 1000 births in 2005 (a 2.0\% [95\% CI, $1.6 \%-2.4 \%$ ] increase in odds per year), although there was a plateau from 2001 onwards (Box 1). When the analysis was restricted to mothers with a current mental health contact, there was an increase from 12 per 1000 births in 1990 to 27 per 1000 births in 2005 (4.7\% [95\% CI, 4.2\%-5.2\%] increase in odds per year) for all births, and an increase from 14 per 1000 births in 1990 to 17 per 1000 births in 2005 (2.4\% [95\% CI, $1.6 \%-3.3 \%$ ] increase in odds per year) for first-born children (Box 1).

There were 31201 children born to fathers with a prior mental health disorder. Overall, prevalence of prior mental health disorders in fathers increased, from 56 per 1000 births in 1990 to 88 per 1000 births in 2005 (Box 1). There was a $3.1 \%(95 \% \mathrm{CI}, 2.8 \%-3.4 \%)$ increase per year in the odds of children being born to fathers with a prior mental health disorder. Prevalence of prior mental health disorders in fathers of first-born children increased at a similar rate, from 52 per 1000 births in 1990 to 71 per 1000 births in 2005 (a 2.4\% [95\% CI, 2.0\%-2.8\%] increase in odds per year). However, similar to mothers, the rate plateaued from 2000 onwards. When the analysis was restricted to fathers with a current mental health contact, the rates were 7.9 per 1000 births in 1990 and 8.2 per 1000 births in 2005 for all births, with fluctuations over that time (Box 1). 
2 Prevalence of children having a parent with a prior mental health disorder, by mental health diagnostic group (all births, Western Australia, 1990-2005)

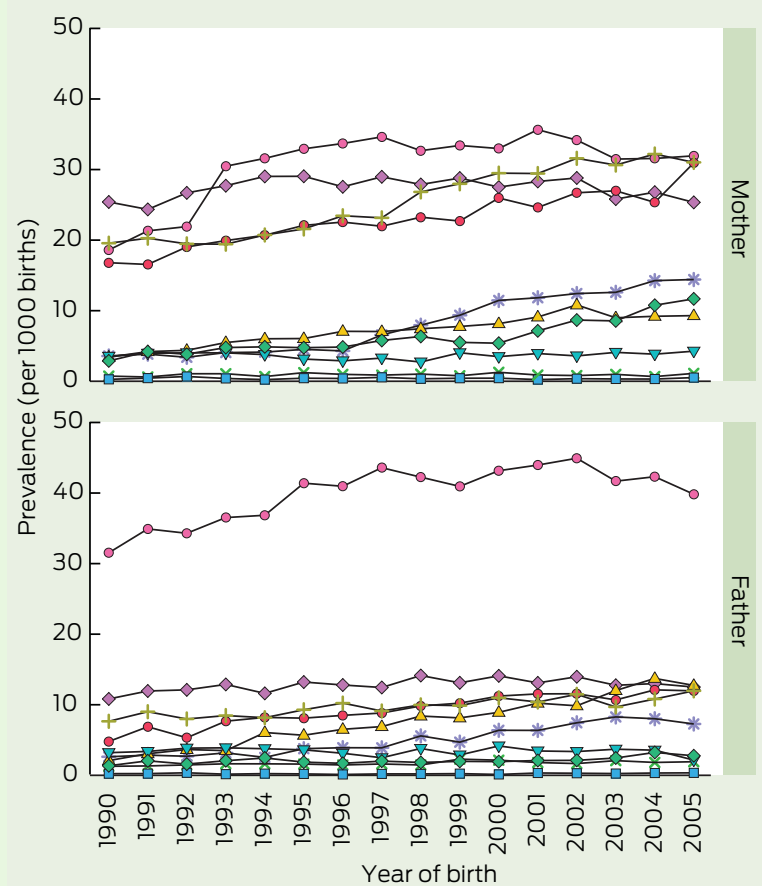

- Adjustment and stress-related disorders

$\triangle$ Disorders of childhood

+ Depression not elsewhere classified and neurotic disorders

$\times$ Organic disorders

$\diamond$ Other mental disorders

$\checkmark$ Personality disorders

- Mental retardation

* Non-organic psychotic disorders

Self-harm ever

- Substance-related disorders adjustment and stress-related disorders (5.5\% per year) and non-organic psychotic disorders $(16.7 \%$ per year). The most prevalent disorders among fathers at any time before birth were substancerelated disorders, which increased from 32 per 1000 births to 40 per 1000 births (1.7\% increase per year; Box 2). Among fathers, there were also large increases in organic disorders (6.7\% per year) and adjustment and stress-related disorders ( $9.3 \%$ per year).

The proportion of Aboriginal children born to mothers with a prior mental health disorder (269 per 1000 births) was more than twice that for non-Aboriginal children (119 per 1000 births) in 2005. The rates were lower for fathers, but there was a similar difference between Aboriginal children (204 per 1000 births) and non-Aboriginal children (80 per 1000 births). The rates for Aboriginal and non-Aboriginal children had increased from 1990 to 2005, although the increases were greater for Aboriginal children $(5.8 \%$ v $3.3 \%$ increase in odds per year for mothers; $6.5 \%$ v $2.6 \%$ increase in odds per year for fathers).

There was a higher prevalence of mental health disorders in mothers and fathers aged $<20$ years in 2005 compared with the other age groups (158 per 1000 births and 147 per 1000 births, respectively), closely followed by those aged 20-29 years (148 per 1000 births and 114 per 1000 births, respectively).

The results of a multiple logistic regression analysis of factors associated with the odds of the first child being born to a mother with a prior mental health disorder are shown in Box 3. Young maternal age ( $<30$ years), low socioeconomic status and being Aboriginal significantly increased the odds.

3 Factors associated with the first child being born to a mother with a prior mental health disorder

\begin{tabular}{lcccc} 
& \multicolumn{4}{c}{ Odds ratio $(95 \% \mathrm{Cl})$} \\
\cline { 2 - 5 } & $1990-1993$ & $1994-1997$ & $1998-2001$ & $2002-2005$ \\
\hline Aboriginal or Torres Strait Islander & $1.3(1.1-1.5)$ & $1.2(1.0-1.4)$ & $1.2(1.1-1.4)$ & $1.2(1.1-1.4)$ \\
Maternal age & & & & \\
$<20$ years & $1.9(1.6-2.2)$ & $1.7(1.5-2.0)$ & $2.1(1.9-2.4)$ & $2.2(1.9-2.5)$ \\
$20-29$ years & $1.2(1.1-1.4)$ & $1.2(1.1-1.3)$ & $1.3(1.2-1.5)$ & $1.4(1.3-1.6)$ \\
$30-39$ years & Reference & Reference & Reference & Reference \\
$>39$ years & $1.2(0.7-1.9)$ & $1.0(0.6-1.4)$ & $1.4(1.0-1.8)$ & $1.2(0.9-1.6)$ \\
Socioeconomic status & & & & \\
$1-$ low disadvantage & Reference & Reference & Reference & Reference \\
2 & $1.1(1.0-1.3)$ & $1.3(1.2-1.5)$ & $1.2(1.1-1.4)$ & $1.1(1.0-1.2)$ \\
3 & $1.2(1.0-1.3)$ & $1.4(1.2-1.6)$ & $1.4(1.3-1.6)$ & $1.3(1.2-1.5)$ \\
$4-$ high disadvantage & $1.5(1.3-1.7)$ & $1.7(1.5-1.9)$ & $1.7(1.5-1.9)$ & $1.6(1.5-1.8)$
\end{tabular}

An increasing temporal trend in the odds of being born to a mother with a prior mental health disorder was seen in the youngest maternal age category ( $<20$ years)

\section{Discussion}

Our study is one of few that have used population-level linked data to determine the prevalence of a prior history of mental health disorders in parents and, to our knowledge, the only study to examine trends over time. It shows that prevalence of prior mental health disorders increased in parents of infants born from 1990 to 2005; there was a $3.7 \%$ increase per year for mothers and a $3.1 \%$ increase per year for fathers. The lower prevalence for fathers could be due to underascertainment of fathers on the Birth Register, which has been documented previously. ${ }^{11}$

There are a number of possible reasons for the increase. It could be due to broader service availability in WA for inpatients and outpatients, the increasing trend to deinstitutionalise people with mental health disorders (particularly during the mid 1980s), ${ }^{12}$ and better data collection by the Mental Health Information System. However, there could be a true increase in the prevalence of mental health disorders in parents. A previous study by our group showed that there had been an increase in the prevalence of babies born with neonatal withdrawal syndrome in $\mathrm{WA}^{13}$ indicating increasing drug use by pregnant women. This is also indicated by our data, which show increasing trends in mental health disorders relating to substance use between 1990 and 2005. Another study that used linked prescription and birth data has shown high and increasing use of antidepressants during pregnancy. ${ }^{14}$ An Australian national survey of psychosis also showed an increase (from 1997 to 2010) in the number of dependent children living at home with parents who have psychosis, particularly mothers. ${ }^{15}$ These data highlight the increasing burden of parental mental health disorders on the mental health system and on families.

The diagnostic group for which prevalence was highest, in mothers and fathers, was substance-related disorders. For mothers, prevalence was also high for three other diagnostic groups: self-harm ever; depression not otherwise classified and neurotic disorders; 
and adjustment and stress-related disorders. Aboriginal children had a greater burden of parental mental health disorders compared with non-

Aboriginal children for both mothers and fathers. This is consistent with findings from a national survey which found that Aboriginal Australians were twice as likely to report high or very high levels of psychological distress compared with non-Aboriginal Australians. ${ }^{16}$ In addition, our study shows that there were larger increases over time in the proportions of Aboriginal children born to mothers and fathers with a prior mental health disorder compared with increases for non-Aboriginal children. This indicates an increasing burden in Aboriginal families of parents with a mental disorder, and highlights the need for culturally appropriate mental health services such as healing and wellbeing centres, as called for in the Bringing them home report. ${ }^{17}$

Younger parental age is also associated with a higher burden of mental health disorders and this has risen over time. National mental health surveys have shown that young people aged 16-24 years have the highest prevalence of mental health disorders in the 12 months before completing the survey, ${ }^{6}$ which is consistent with our findings.

Although the strengths of our study included its population-based nature and the long period of data collection, our data on prevalence of mental health disorders are likely to be underestimates because we only captured inpatient hospital admissions and public outpatient contacts in the Mental Health Information System. We did not have access to primary care data or a prescription database that could have provided data on individuals who saw general practitioners or received prescriptions for mental health disorders; therefore, we may have potentially missed patients in the community with mental health disorders. By only using hospital admissions data and public outpatient contact data, our sample is biased towards patients with more severe mental disorders.

Despite these limitations, the use of population-level data overcomes many of the difficulties associated with traditional research designs, such as surveys or interviews. Parents may be reluctant to admit to a mental health disorder, particularly those related to substance use. It is important to obtain prevalence estimates of parental mental health disorders to inform practitioners, service providers and policymakers on the extent of the burden and the resources required. Most importantly, our study shows that there has been an increase in prevalence of children born to parents with previous mental health contacts. Moreover, although underascertained, our study includes fathers, an important group who are often not captured or not assessed in surveys and screening tools. Our study also highlights high-risk groups that have had increasing mental health admissions over time, including Aboriginal families, younger parents and low socioeconomic groups. These high-risk groups should be a focus for intervention and prevention.

In terms of practice and policy implications, parents with mental health disorders should be offered early intervention, referral and support. The National Perinatal Depression Initiative has highlighted the importance of early assessment and intervention in the perinatal period. ${ }^{18}$ GPs and mental health workers also play important roles in discussing the ongoing impact of mental health disorders on families and in working with families to plan for respite and support when required. The National Institute for Health and Clinical Excellence has provided guidelines for the clinical management of antenatal and postnatal mental health problems experienced by mothers and has stipulated the importance of the ongoing management of mental disorders. ${ }^{19}$ As the World Health Organization has stated, "effective solutions for mental disorders are available ... Some mental disorders can be prevented, while most can be treated". ${ }^{20}$ Mental health needs to be considered during treatment and health care planning for parents, to alleviate the symptoms experienced by individuals and to reduce the impact of symptoms on children and their families.

Acknowledgements: This work was supported by an Australian Research Council (ARC) Linkage Project grant (LP100200507) and an Australian Research Council Discovery Project grant (DP110100967). We acknowledge the partnership of the WA Department of Health Department fo Child Protection, Department of Education, Disability Services Commission, Department of Corrective Services and Department of the Attorney General who provided support as well as data for this project. This article does not necessarily reflect the views of the government departments involved in this research. Melissa O'Donnell is supported by a National Health and Medical Research Council (NHMRC) Early Career Fellowship (1012439). Natasha Nassar is supported by a NHMRC Career Development Award (632955). We also thank the WA Data Linkage Branch for linking the data.
Competing interests: No relevant disclosures.

Received 7 Dec 2012, accepted 17 Apr 2013.

1 Rutter M, Quinton D. Parental psychiatric disorder: effects on children. Psychol Med 1984; 14: 853-880.

2 Smith M. Parental mental health: disruptions to parenting and outcomes for children. Child Fam Soc Work 2004; 9: 3-11.

3 Huntsman L. Parents with mental health issues: consequences for children and effectiveness of interventions designed to assist children and their families. Sydney: Centre for Parenting and Research, NSW Department of Community Services, 2008.

4 Australian Institute of Health and Welfare. Child protection Australia 2010-11. Canberra: AlHW, 2012. (AlHW Cat. No.CWS 41; Child Welfare Series No. 53.)

5 Victorian Government Department of Human Services. An integrated strategy for child protection and placement services. Melbourne: DHS, 2002.

6 Australian Bureau of Statistics. National Survey of Mental Health and Wellbeing: summary of results, 2007. Canberra: ABS, 2008.

7 World Health Organization. International classification of diseases, ninth revision. Geneva: WHO, 1979.

8 Holman CD, Bass AJ, Rouse IL, Hobbs MS. Population-based linkage of health records in Western Australia: development of a health services research linked database. Aust NZ J Public Health 1999; 23: 453-459.

9 Australian Bureau of Statistics. Socio-economic Indexes for Areas (SEIFA) - technical paper, 2006. Canberra: ABS, 2008

10 RDevelopment Core Team. R: a language and environment for statistical computing. Vienna: R Foundation for Statistical Computing, 2010.

11 Morgan V, Croft M, Valuri G, et al. Intellectual disability and other neuropsychiatric outcomes in high-risk children of mothers with schizophrenia, bipolar disorder and unipolar major depression. Br J Psychiatry 2012; 200: 282-289.

12 Burdekin B, Guilfoyle M, Hall D. Human rights and mental illness. Report of the National Inquiry into the Human Rights of People with Mental Illness. Canberra: Australian Govemment Publishing Service, 1993.

13 O'Donnell M, Nassar N, Leonard H, et al. Increasing prevalence of neonatal withdrawal syndrome: population study of maternal factors and child protection involvement. Pediatrics 2009; 123 : e614-e621.

14 Colvin L, Slack-Smith L, Stanley FJ, et al. Dispensing patterns and pregnancy outcomes for women dispensed selective serotonin reuptake inhibitors in pregnancy. Birth Defects Res A Clin Mol Teratol 2011; 91: 142-152

15 Morgan VA, Waterreus A, Jablensky A, et al. People living with psychotic illness in 2010: the second Australian national survey of psychosis. Aust N Z Psychiatry 2012; 46: 735-752.

16 Australian Institute of Health and Welfare. Measuring the social and emotional wellbeing of Aboriginal and Torres Strait Islander peoples. Canberra: AlHW, 2009.

17 Human Rights and Equal Opportunity Commission. Bringing them home: report of the National Inquiry into the Separation of Aboriginal and Torres Strait Islander Children from their Families. Sydney: Human Rights and Equal Opportunity Commission, 1997.

18 beyondblue; Perinatal Mental Health Consortium Perinatal Mental Health National Action Plan 2008-2010: full report. Melbourne: beyondblue, 2008 .

19 National Institute for Health and Clinical Excellence. Antenatal and postnatal mental health: clinical management and service guidance. London: National Institute for Health and Clinical Excellence, 2007.

20 World Health Organization. The world health report 2001 - mental health: new understanding, new hope.Geneva:WHO, 2001: 109 
Research 